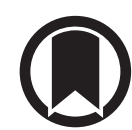

CrossMark

\section{Rapid onset honeycombing fibrosis in spontaneously breathing patient with COVID-19}

To the Editor:

A 38-year-old man presented with a 6-day history of gradually worsening cough and asthenia and secondary anosmia and agueusia. He had no prior personal medical history. His mother had a history of diabetes mellitus and his son was followed for haemophilia A. His spouse had exhibited similar symptoms a week earlier.

On admission to the emergency room, clinical examination revealed crackles in the right lung, tachypnoea with a respiratory rate of 40 per min, tachycardia with a pulse of 119 beats per minute, an oxygen saturation of $66 \%$ while breathing ambient air. Body temperature was $39.8^{\circ} \mathrm{C}$ and blood pressure $117 /$ $72 \mathrm{mmHg}$. Initial computed tomographic pulmonary angiography (CTPA) showed bilateral ground-glass opacities, condensation in the right lower lobe, and no sign of pulmonary embolism (figure 1a). Nasal swab for severe acute respiratory syndrome coronavirus 2 (SARS-CoV-2) testing was obtained and result of the RT-PCR testing was positive.

The patient was promptly transferred to the intensive care unit, where oxygen therapy with high flow nasal cannula was initiated with an oxygen outflow of $50 \mathrm{~L} \cdot \mathrm{min}^{-1}$ and a fraction of inspired oxygen $\left(F_{\mathrm{IO}_{2}}\right)$ of 1 initially, along with an empirical course of antibiotics (ceftriaxone and levofloxacin). A trial of prone therapy was started, yielding immediate positive results. While in prone position, the patient was no longer tachypnoeic, and required lower levels of oxygen support, allowing us to delay initiating mechanical ventilation.

Over the next few days, prone positioning was regularly performed. Although arterial oxygen tension $\left(P_{\mathrm{aO}_{2}}\right)$ levels were around $75 \mathrm{mmHg}$ on an $F_{\mathrm{IO}_{2}}$ of 0.8 , corresponding to a Horowitz $\left(P_{\mathrm{aO}_{2}} / F_{\mathrm{IO}_{2}}\right)$ ratio of 90 , the patient did not exhibit any clinical signs of respiratory distress, no shortness of breath nor tachypnoea. His ROX index $\left(\left(S_{\mathrm{pO}_{2}} / F_{\mathrm{IO}_{2}}\right) /\right.$ respiratory rate) [1] remained steadily superior to 5. Considering the above, mechanical ventilation was not initiated. Fever quickly receded, and inflammatory marker levels decreased. Antibiotics were discontinued on day 4 because of lack of evidence of any secondary bacterial infection.

On day 10, because the patient still required high levels of oxygen via high-flow nasal cannula, a control CTPA was performed (figure 1b). It showed no evidence of pulmonary embolism, but rapid onset of extensive pulmonary honeycombing fibrosis in the territories where ground glass opacities had been present. Multidisciplinary meeting concluded that rapid onset pulmonary fibrosis complicated the course of severe coronavirus disease 2019 (COVID-19) pneumonia. Search for other causes of interstitial lung disease, including autoimmune disease, was negative. Treatment included daily high-dose intravenous corticosteroids boluses of $250 \mathrm{mg}$ for 3 days, followed by $1 \mathrm{mg}$ per $\mathrm{kg}$ per day, associated with antifibrotic therapy with nintedanib. The patient's condition improved and he was transferred to pneumology wards on day 18 on oxygen therapy $\left(6 \mathrm{~L} \cdot \mathrm{min}^{-1}\right)$.

Angiotensin-converting enzyme 2 (ACE-2), identified as the SARS-CoV-2 receptor, is expressed in different pulmonary cell types. Injury of type II pneumocytes combined with an excessive and aberrant host immune and/or repair responses observed in COVID-19 could induce pulmonary fibrosis of variable severity, the prevalence of which remains to be determined in large prospective follow-up of COVID-19 cohorts. The other targets of SARS-CoV-2 are pulmonary endothelial cells, resulting in pulmonary

@ERSpublications

Patients with COVID-19 should be monitored for rapid onset honeycombing fibrosis https://bit.ly/ 2B7fRcA

Cite this article as: Combet $\mathrm{M}$, Pavot $\mathrm{A}$, Savale L, et al. Rapid onset honeycombing fibrosis in spontaneously breathing patient with COVID-19. Eur Respir J 2020; 56: 2001808 [https://doi.org/10.1183/ 13993003.01808-2020]. 
a)
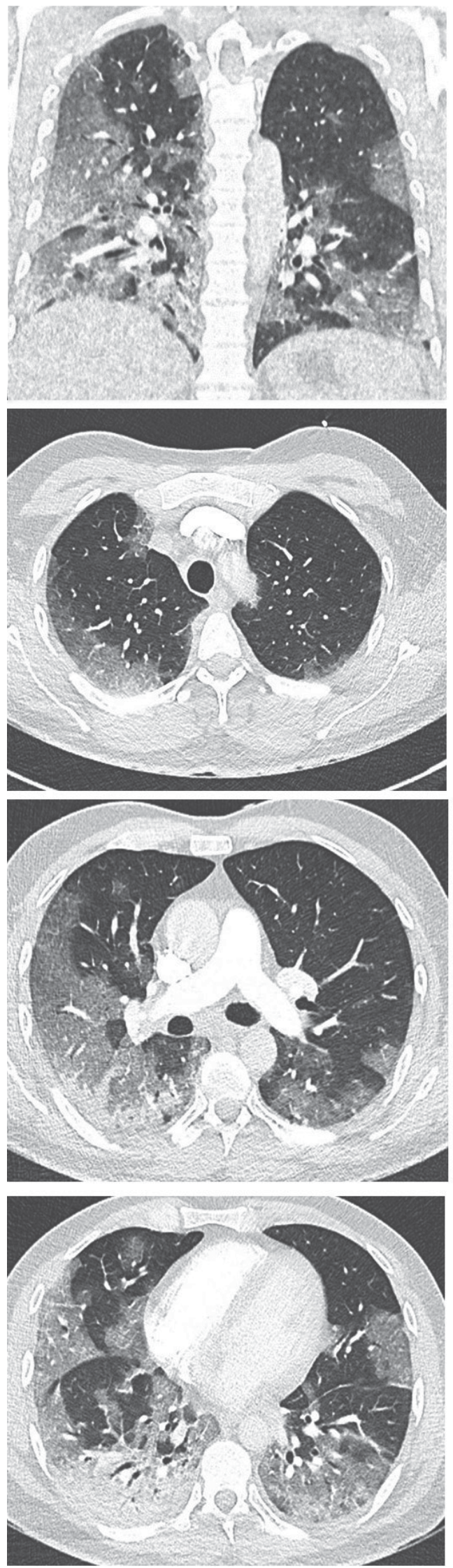

b)
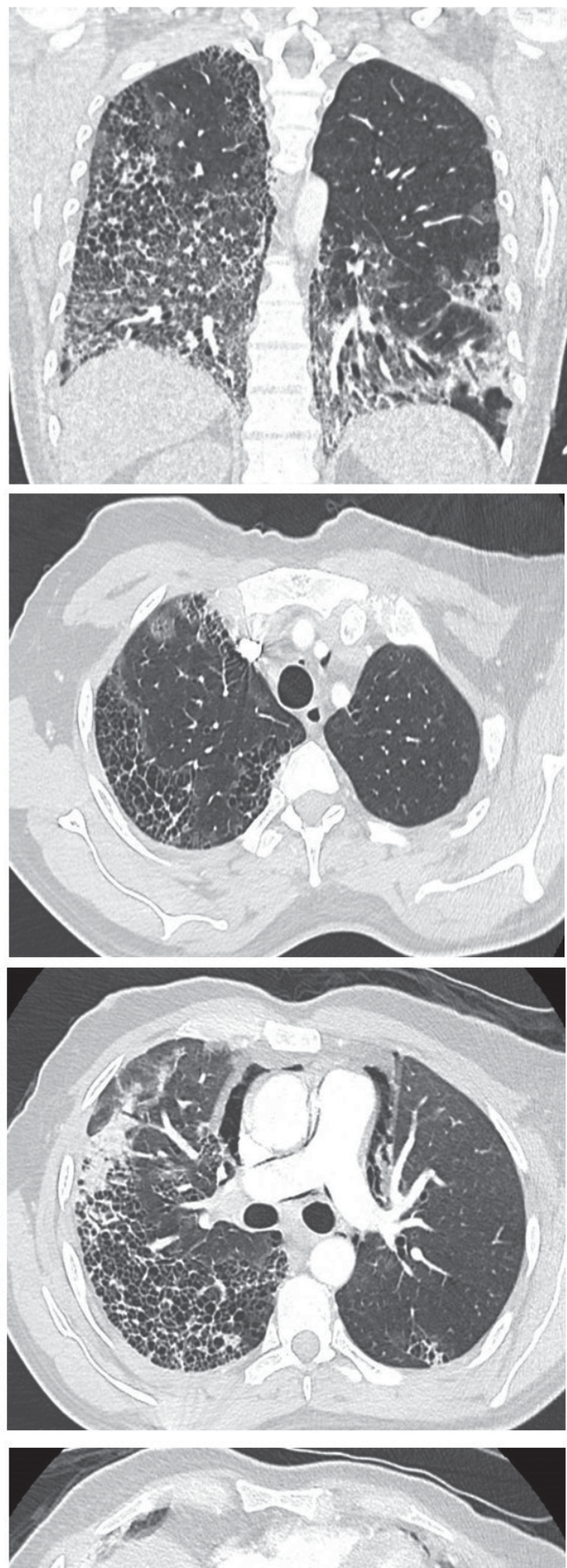

a)

1.

- 989

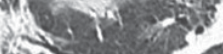

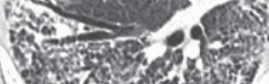

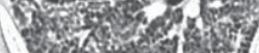

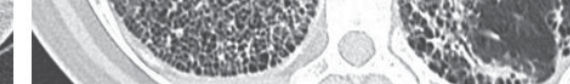


FIGURE 1 a) Initial CT scan: images show extensive ground-glass opacities in both lungs with subpleural predominance affecting $50-75 \%$ of lung parenchyma, as well as condensation in the right lower lobe. b) Control CT imaging at day-10 : axial CT images show extensive honeycombing cysts associated with septal thickening, with subpleural predominance (where ground-glass opacities had initially been observed, notably in the right lower and upper lobes), with associated traction bronchiectasis. Pneumomediastinum also developed. There was no sign of pulmonary embolism.

vascular features as previously described [2]. To our knowledge, few cases of rapid-onset pulmonary fibrosis following SARS-CoV-2 infection have been reported so far. CHEN et al. [3] reported three cases of lung transplantation for post-acute respiratory distress syndrome (ARDS) fibrosis due to SARS-CoV-2, but all three patients had been undergoing mechanical ventilation for over 3 weeks for severe ARDS before transplantation. To our knowledge, the present case is the first to describe rapid onset extensive pulmonary fibrosis in a COVID-19 patient who never required mechanical ventilation. The pattern of lung fibrosis and the speed of its development is atypical in comparison with the usual fibrotic response following lung injury, suggesting that the combination of the direct effect of the virus on pulmonary alveolar and endothelial cells, combined with the aberrant local immune response might induce aggressive lung fibrosis in predisposed patients.

Margot Combet ${ }^{1}$, Arthur Pavot $\oplus^{1,2}$, Laurent Savale $\oplus^{2,3,4}$, Marc Humbert $\oplus^{2,3,4}$ and Xavier Monnet ${ }^{1,2,4}$

${ }^{1}$ AP-HP, Service de médecine intensive-réanimation, Hôpital de Bicêtre, Le Kremlin-Bicêtre, France. ${ }^{2}$ Université ParisSaclay, Faculté de Médecine, Le Kremlin-Bicêtre, France. ${ }^{3}$ AP-HP, Service de pneumologie et soins intensifs respiratoires, Centre de Référence de l'Hypertension Pulmonaire, Hôpital de Bicêtre, Le Kremlin-Bicêtre, France. ${ }^{4}$ INSERM UMR_S 999, Hôpital Marie-Lannelongue, Le Plessis-Robinson, France.

Correspondence: Arthur Pavot, AP-HP, Service de médecine intensive-réanimation, Hôpital de Bicêtre, 63 rue Gabriel Péri, Le Kremlin-Bicetre, 94275, France. E-mail: pavot.arthur@gmail.com

Received: 15 May 2020 | Accepted after revision: 21 June 2020

Conflict of interest: M. Combet has nothing to disclose. A. Pavot has nothing to disclose. L. Savale reports personal fees and non-financial support from Actelion and MSD, grants, personal fees and non-financial support from GSK, outside the submitted work. M. Humbert reports personal fees from Novartis, during the conduct of the study; grants, personal fees and non-financial support from GlaxoSmithKline, personal fees from AstraZeneca, Novartis, Roche, Sanofi and Teva, outside the submitted work. X. Monnet has nothing to disclose.

\section{References}

1 Roca O, Messika J, Caralt B, et al. Predicting success of high-flow nasal cannula in pneumonia patients with hypoxemic respiratory failure: the utility of the ROX index. J Crit Care 2016; 35: 200-205.

2 Ackermann M, Verleden SE, Kuehnel M, et al. Pulmonary vascular endothelialitis, thrombosis, and angiogenesis in Covid-19. N Engl J Med 2020; 383: 120-128.

3 Chen J-Y, Qiao K, Liu F, et al. Lung transplantation as therapeutic option in acute respiratory distress syndrome for COVID-19-related pulmonary fibrosis. Chin Med J 2020; 133: 1390-1396. 